\title{
Antiferromagnetic Spin Ladders with Odd and Even number of Chains
}

\author{
Asimkumar Ghosh and Indrani Bose \\ Department of Physics, \\ Bose Institute, \\ 93/1, Acharya Prafulla Chandra Road, \\ Calcutta-700 009, India.
}

\begin{abstract}
We construct frustrated antiferromagnetic spin ladders with $\mathrm{m}$ chains for which the exact ground state can be determined in a particular parameter regime. The excitation spectrum is shown rigorously to be gapless ( with gap ) for odd ( even ) m. In a general parameter regime, the four-chain and periodic ladders are studied using a meanfield theory based on the bond operator formalism for spin $S=\frac{1}{2}$. The excitation spectrum and the spin-gap are calculated in both the cases. The spin-gap of the frustrated ladder system has a larger magnitude than in the case of non-frustrated ladders. For the frustrated periodic ladder, the spin-gap vanishes at a critical value of the interladder coupling strength which is larger than the critical value in the case of non-frustrated periodic ladder.
\end{abstract}

P.A.C.S. NO: $75.10 . J m$ 


\section{Introduction}

Antiferromagnetic (AFM) spin $\mathrm{S}=1 / 2$ ladders have been extensively studied in recent times as the ladder interpolates between the $1 \mathrm{~d}$ chain and $2 \mathrm{~d}$ plane. [1] The $\mathrm{S}=1 / 2$ Heisenberg AFM chain is exactly solvable using the wellknown Bethe Ansatz. [2] The ground state is disordered and characterised by a power law decay of the two-spin correlation function. The excitation spectrum is known to be gapless. The planar (square lattice) $\mathrm{S}=1 / 2 \mathrm{AFM}$ with nearest-neighbour coupling shows long range AFM order at zero temperature and the excitation spectrum is gapless.

The copper-oxide planes of the high- $T_{c}$ cuprate systems in the undoped state serve as good examples of $2 \mathrm{~d}$ AFMs defined on the square lattice. This fact has sparked renewed interest in the study of low-dimensional AFMs. One particularly interesting problem is to study the crossover from AFM chains to the square lattice. The crossover can be understood by examining n-chain spin ladders with increasing width. Such studies have yielded the surprising result that for odd (even) values of $n$, the excitation spectrum is gapless (with gap). Ladders consisting of an even number of chains have spin liquid ground state with exponential decay of the two-spin correlation function. The spin 1 excitation of the ladder has a finite energy gap. A ladder with an odd number of chains has quite different behaviour and displays characteristics similar to those of single chains, namely, gapless spin excitations and a power-law decay of the two-spin correlations. The significant difference between even-chain and odd-chain ladders can be attributed to quantum effects.

The compound $(\mathrm{VO})_{2} \mathrm{P}_{2} \mathrm{O}_{7}$ has a two-chain ladder configuration of spin$1 / 2 V^{4+}$ ions. [3] Real compounds like stoichiometric $\mathrm{Sr}_{n-1} \mathrm{Cu}_{n+1} \mathrm{O}_{2 n},(n=$ $3,5,7,9, \ldots)$, 4 can be described by m-chain spin ladders with $m=\frac{n+1}{2}$. 
Spin susceptibility and nuclear magnetic resonance (NMR) experiments on the two-chain ladder systems show the existence of a gap in the excitation spectrum. Neutron scattering and muon spin resonance experiments give clear signs of short-range spin order in the 2-chain ladders. [1] Three-chain ladders $\left(\mathrm{Sr}_{2} \mathrm{Cu}_{3} \mathrm{O}_{5}\right)$ by contrast show longer range spin correlations and a gapless spectrum. There is also true long range order at low temperatures

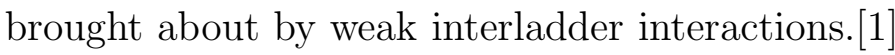

Some theoretical studies have been undertaken recently to understand the 'odd-even' effect of spin ladders. Reigrotzki et al [5] have studied the properties of spin ladders with two, three, and four chains expanded in the ratio of the intrachain and interchain coupling constants. Khveshchenko 6] has shown that for odd-chain ladders a topological term appears in the effective action corresponding to the dynamics at long wavelengths. For even-chain ladders there is no such term. This topological term is similar to the one responsible for the difference between integer and half-odd integer spin chains. Integer spin chains have a gap ( the Haldane gap ) in the excitaion spectrum whereas half-odd integer spin chains are gapless. Recent studies [7, 8] have shown that two-chain spin ladders with both ferromagnetic and antiferromagnetic rung exchange interactions are Haldane gap systems in the appropriate limits. Rojo [9] has further given a rigorous proof for the absence of gap for spin 1/2 ladders with an odd number of chains in the infinite chain length limit.

In this paper, we construct models of spin ladders with odd and even number of chains for which the exact ground states can be determined and the 'odd-even' effect associated with the excitation spectrum of ladders can be demonstrated rigorously. In Sec. II, a description of the ladders is given 
and the ground and first excited state determined. In Sec. III, the properties of a four-chain ladder are studied using a mean field theory based on the bond operator formalism [4, 10]. The calculations are further extended to periodic ladders. Sec. IV contains concluding remarks.

\section{Model spin ladders}

Bose and Gayen [11, 12, 13] have constructed a two-chain spin ladder model for which several exact results can be derived both in the undoped and holedoped states. The ladder is shown in Fig. 1. Every site is occupied by a spin of magnitude $1 / 2$. The spins interact with Heisenberg AFM exchange interaction. The nearest-neighbour (n.n.) intra-chain exchange interaction is of strength $\beta$, the rung and n.n. diagonal exchange interactions are of strength $\alpha$ and $\gamma$ respectively. For $\beta=\gamma$ and $\frac{\alpha}{\beta} \geq 2$, Bose and Gayen [1] showed that the exact ground state consists of singlet spin configurations $\left(\frac{1}{\sqrt{2}}(\uparrow \downarrow-\downarrow \uparrow)\right)$ along the rungs of the ladder. We construct spin ladders of increasing width by adding chains and stipulating that alternate two-chain ladders have no diagonal exchange inteactions. The spins located in the rungs of the model interact via the 'sawtooth-chain' interaction. Such a chain is illustrated in Fig. 2. In Figs. 3(a) and 3(b), the spin ladders with three and four chains respectively are shown. For both odd and even-chain spin ladders one can write down an exact eigenstate which is also the exact ground state in an appropriate parameter regime. The exact eigenstate for an even-chain spin ladder consists of spin singlets along the rungs of two-chain ladders with diagonal exchange interactions. The proof of eigenstate can be easily obtained using the spin identity $\vec{S}_{n} \cdot\left(\vec{S}_{l}+\vec{S}_{m}\right)[l m] \equiv 0$ where [lm] 
describes a spin singlet of spins at sites 1 and $\mathrm{m}$. Let $\eta$ be the strength of the exchange interaction between the next-nearest-neighbour (n.n.n.) spins along the rungs. The rung exchange interactions for two chain ladders without diagonal interactions are of strength $\xi$. Consider the parameter regime $\beta=$ $\gamma=\xi=\eta$ and $\frac{\alpha}{\beta} \geq 3$. In this regime, the exact eigenstate described before becomes the exact ground state. The proof of the exact ground state can be obtained by using the well-known method of 'divide and conquer'. [11] Let $E_{1}$ be energy of the exact eigenstate and $E_{G}$ the energy of the exact ground state. Then $E_{G} \leq E_{1}$. For the specified eigenstate $E_{1}=\frac{-3 N \alpha}{4}$ where $\mathrm{N}$ is the total number of n.n. vertical bonds (rungs) in the system along which singlets form in the ground state. The total spin Hamiltonian $\mathrm{H}$ can be divided into two parts $H_{L}$ and $H_{S} . H_{L}$ describes the exchange interactions of all the twochain ladders in the system with diagonal interactions. Each such ladder has exchange interaction strengths $\alpha_{1}\left(\alpha_{1} \geq 2 \beta\right), \beta$ and $\beta$ for the rung, intra-chain n.n. and diagonal interactions respectively. The exact ground state energy $E_{L}=\frac{-3 N \alpha_{1}}{4} . H_{S}$ corresponds to all the rung interactions in the system having the geometrical structure of sawtooth chains. All the spinspin interactions in the sawtooth are of strength $\beta$. The ground state energy $E_{S}=\frac{-3 N \beta}{4}$. Let $\Psi_{G}$ be the exact ground state wave function. Then by variational theorem,

$$
\begin{aligned}
& \qquad\left\langle\Psi_{G}|H| \Psi_{G}\right\rangle=E_{G}=\left\{\left\langle\Psi_{G}\left|H_{L}\right| \Psi_{G}\right\rangle+\left\langle\Psi_{G}\left|H_{S}\right| \Psi_{G}\right\rangle\right\} \\
& \text { i.e., } E_{1} \geq E_{G} \geq E_{L}+E_{S} \text {. } \\
& \text { Now, } E_{1}=\frac{-3 N \alpha}{4}\left(\alpha=\alpha_{1}+\beta \geq 3 \beta\right)=E_{L}+E_{S} \text {. So } E_{1}=E_{G}
\end{aligned}
$$
and the exact eigenstate is also the exact ground state. For a spin ladder of odd number of chains, all the chains except one belong to the two-chain 
ladders with diagonal exchange interactions. Again, in the parameter regime $\alpha \geq 3 \beta$ and $\beta=\gamma=\xi=\eta$, the exact ground state consists of spin singlets along the rungs of the two-chain ladders with diagonal interactions and the isolated chain has spin configuration corresponding to that of the Heisenberg AFM chain. The proof of exact ground state is similar to that for even-chain ladders.

We now consider the excited states of the system. For odd-chain ladders, the lowest lying excited state is the triplet $(S=1)$ excitation of the HAFM chain. The excitation energy is given by 15

$$
\omega=\frac{\pi}{2} \beta|\sin (q)|
$$

where $\mathrm{q}$ is the momentum wave vector w.r.t that of the chain ground state. The excitation is confined to the chain which does not belong to the twochain ladders with diagonal couplings. The spectrum is gapless for $q=0$ and $\pi$. In the spin-ladder systems considered, periodic boundary condition is assumed to hold true in the horizontal (x) direction. The ladder has infinite length in this direction. For the two-chain spin ladder shown in Fig. 1 and in the parameter regime under consideration, the lowest excited states consist of a triplet along one of the rungs. The excitation energy measured w.r.t the ground state energy is $\alpha$ which is a measure of the spin-gap. The triplet excitation is localised and has no dynamics in the x-direction. For an evenchain ladder, the spin dynamics is only in the vertical y-direction and the lowest excited state corresponds to that of the sawtooth chain. Consider the four-chain ladder shown in Fig. 3(b). Exact diagonalisation of four-sited sawtooth chain shows that the first excited state has energy $-\frac{(1+\sqrt{33})}{4} \beta$. The energy measured w.r.t the ground state energy, $-\frac{18 \beta}{4}$, gives a spin-gap which is less than $\alpha=3 \beta$, the spin gap for the two-chain ladder. The spin-gap thus 
decreases in magnitude as the number of chains in the even-chain ladder increases from two to four. In the next section, we consider more general parameter regimes in which the exact ground and excited states are not known. We study the even-chain ladders only and determine the excitation spectrum and spin-gap for both the four-chain and periodic ladders.

\section{Four-chain and periodic ladder}

The properties of a two-chain spin ladder are already known in the mean-field theory. [16] The Hamiltonian is given by (Fig.1)

$$
\begin{aligned}
H & =\sum_{i}\left\{\alpha \mathbf{S}_{i} \cdot \mathbf{S}_{i}^{\prime}+\beta\left(\mathbf{S}_{i}^{\prime} \cdot \mathbf{S}_{i+1}^{\prime}+\mathbf{S}_{i} \cdot \mathbf{S}_{i+1}\right)\right. \\
& \left.+\gamma\left(\mathbf{S}_{i} \cdot \mathbf{S}_{i+1}^{\prime}+\mathbf{S}_{i}^{\prime} \cdot \mathbf{S}_{i+1}\right)\right\}
\end{aligned}
$$

The ground state is assumed to be in a dimerized phase with the singlet dimers located along the rungs. The bond operator representation of $\mathbf{S}=1 / 2$ spins is used to study the properties of dimerized phases. We consider two spins $(\mathrm{S}=1 / 2) S_{i}^{\prime}$ and $S_{i}$ placed on each rung. The Hilbert space consists of four states which in appropriate combinations describe the singlet $|\mathbf{s}\rangle$ and the three triplet $\left|\mathbf{t}_{\mathbf{x}}\right\rangle,\left|\mathbf{t}_{\mathbf{y}}\right\rangle$ and $\left|\mathbf{t}_{\mathbf{z}}\right\rangle$ states. These states are created out of the vacuum $|0\rangle$ by the singlet and triplet creation operators

$$
\begin{aligned}
& |\mathbf{s}\rangle=\mathbf{s}^{\dagger}|0\rangle=\frac{1}{\sqrt{2}}(|\uparrow \downarrow\rangle-|\downarrow \uparrow\rangle) \\
& \left|\mathbf{t}_{x}\right\rangle=\mathbf{t}_{x}^{\dagger}|0\rangle=-\frac{1}{\sqrt{2}}(|\uparrow \uparrow\rangle-|\downarrow \downarrow\rangle)
\end{aligned}
$$




$$
\begin{aligned}
& \left|\mathbf{t}_{y}\right\rangle=\mathbf{t}_{y}^{\dagger}|0\rangle=\frac{i}{\sqrt{2}}(|\uparrow \uparrow\rangle+|\downarrow \downarrow\rangle) \\
& \left|\mathbf{t}_{z}\right\rangle=\mathbf{t}_{z}^{\dagger}|0\rangle=\frac{1}{\sqrt{2}}(|\uparrow \downarrow\rangle+|\downarrow \uparrow\rangle)
\end{aligned}
$$

The spins $S_{i}^{\prime}$ and $S_{i}$, in terms of the singlet and triplet spin operators, are given by 14,10

$$
\begin{aligned}
& S_{i \alpha}^{\prime}=\frac{1}{2}\left(\mathbf{s}_{\mathbf{i}}^{\dagger} \mathbf{t}_{i \alpha}+\mathbf{t}_{i \alpha}^{\dagger} \mathbf{s}_{\mathbf{i}}-i \epsilon_{\alpha \beta \gamma} \mathbf{t}_{i \beta}^{\dagger} \mathbf{t}_{i \gamma}\right) \\
& S_{i \alpha}=\frac{1}{2}\left(-\mathbf{s}_{\mathbf{i}}^{\dagger} \mathbf{t}_{i \alpha}-\mathbf{t}_{i \alpha}^{\dagger} \mathbf{s}_{\mathbf{i}}-i \epsilon_{\alpha \beta \gamma} \mathbf{t}_{i \beta}^{\dagger} \mathbf{t}_{i \gamma}\right)
\end{aligned}
$$

$\alpha, \beta$ and $\gamma$ are the components along the $\mathrm{x}, \mathrm{y}$ and $\mathrm{z}$ axes respectively and $\epsilon$ is the Levi-Cività symbol and represents the totally antisymmetric tensor. All repeated indices over $\alpha, \beta$ and $\gamma$ are assumed to be summed over.

A constraint of the form

$$
\mathbf{s}^{\dagger} \mathbf{s}+\mathbf{t}_{\alpha}^{\dagger} \mathbf{t}_{\alpha}=1
$$

is assumed to hold true for each dimer so that the physical states can be either singlets or triplets. The singlet and triplet operators at each site satisfy bosonic commutation relations

$$
\left[\mathbf{s}, \mathbf{s}^{\dagger}\right]=1, \quad\left[\mathbf{t}_{\alpha}, \mathbf{t}_{\beta}^{\dagger}\right]=\delta_{\alpha \beta}, \quad\left[\mathbf{s}, \mathbf{t}_{\alpha}^{\dagger}\right]=0
$$

One now substitutes the operator representation of spins given in Eqs.(3.3) and (3.4) into the original Hamiltonian (Eq.(3.1)). A site-dependent chemical potential $\mu_{\mathbf{i}}$ is included in the Hamiltonian to impose the constraint of Eq.(3.5). The transformed Hamiltonian can be solved by a mean-field decoupling of the quartic terms containing two $\mathbf{s}$ and two $\mathbf{t}$ operators as well 
as four $\mathbf{t}$ operators. One takes $\left\langle\mathbf{s}_{i}\right\rangle=\overline{\mathbf{s}}$ and replaces the local constraint $\mu_{\mathbf{i}}$ by a global one $\mu$. One also defines two mean fields as

$$
P=\left\langle\mathbf{t}_{\mathbf{i} \alpha}^{\dagger} \mathbf{t}_{\mathbf{i}+\mathbf{1}, \alpha}\right\rangle \quad Q=\left\langle\mathbf{t}_{\mathbf{i} \alpha} \mathbf{t}_{\mathbf{i}+\mathbf{1} \alpha}\right\rangle
$$

Next, a Fourier transformation of the operators is taken. The resultant Hamiltonian can be diagonalised by the Bogolyubov transformation. Since the details of the calculation are available elsewhere [四] we quote the final results. The diagonalised mean-field Hamiltonian $\mathbf{H}_{\mathbf{m}}(\mu, \overline{\mathbf{s}}, \mathbf{P}, \mathbf{Q})$ is given by

$$
\begin{aligned}
H_{m}(\mu, \overline{\mathbf{s}}, \mathbf{P}, \mathbf{Q}) & =N\left(-\frac{3}{4} \overline{\mathbf{s}}^{2} \alpha-\mu \bar{s}^{2}+\mu\right)-\frac{N}{2}\left(\frac{\alpha}{4}-\mu\right) \\
& -\frac{N \lambda_{2}}{3}\left(P^{2}-Q^{2}\right)+\sum_{k} \omega_{k}\left(\gamma_{k}^{\dagger} \gamma_{k}+\frac{1}{2}\right)
\end{aligned}
$$

where

$$
\begin{aligned}
& \omega_{k}=\sqrt{\Lambda_{k}^{2}-\left(2 \Delta_{k}\right)^{2}} \\
& \Lambda_{k}=\left(\frac{\alpha}{4}-\mu\right)+\left(\lambda_{1} \overline{\mathbf{s}}^{2}+\frac{2 P \lambda_{2}}{3}\right) \cos k \\
& \Delta_{k}=\left(\frac{\lambda_{1} \overline{\mathbf{s}}^{2}}{2}-\frac{Q \lambda_{2}}{3}\right) \cos k \\
& \lambda_{1}=(\beta-\gamma) \\
& \lambda_{2}=(\beta+\gamma)
\end{aligned}
$$

The parameters $\mu, \overline{\mathbf{s}}, \mathbf{P}$ and $\mathbf{Q}$ can be determined from appropriate self- 
consistent equations and the spin-gap $\Delta$ is given by

$$
\Delta=\sqrt{\left(\frac{\alpha}{4}-\mu-\frac{2 \lambda_{2}}{3}(P+Q)\right)\left(\frac{\alpha}{4}-\mu-2 \lambda_{1} \overline{\mathbf{s}}^{2}-\frac{2 \lambda_{2}}{3}(P-Q)\right)}
$$

We now consider the four-chain spin ladder shown in Fig.3(b). The number of two-chain spin ladders is two and they are designated as left (top) and right (bottom) ladders. The two ladders are coupled by exchange interaction of strength $\xi$. The hamiltonian describing the system is given by

$$
\begin{aligned}
H & =\sum_{i}\left\{\alpha\left(\mathbf{S}_{l i} \cdot \mathbf{S}_{l i}^{\prime}+\mathbf{S}_{r i} \cdot \mathbf{S}_{r i}^{\prime}\right)+\beta\left(\mathbf{S}_{l i}^{\prime} \cdot \mathbf{S}_{l i+1}^{\prime}+\mathbf{S}_{l i} \cdot \mathbf{S}_{l i+1}\right.\right. \\
& \left.+\mathbf{S}_{r i}^{\prime} \cdot \mathbf{S}_{r i+1}^{\prime}+\mathbf{S}_{r i} \cdot \mathbf{S}_{r i+1}\right)+\gamma\left(\mathbf{S}_{l i}^{\prime} \cdot \mathbf{S}_{l i+1}+\mathbf{S}_{l i} \cdot \mathbf{S}_{l i+1}^{\prime}\right. \\
& \left.\left.+\mathbf{S}_{r i}^{\prime} \cdot \mathbf{S}_{r i+1}+\mathbf{S}_{r i} \cdot \mathbf{S}_{r i+1}^{\prime}\right)+\eta \mathbf{S}_{l i}^{\prime} \cdot \mathbf{S}_{r i}^{\prime}+\xi \mathbf{S}_{l i} \cdot \mathbf{S}_{r i}^{\prime}\right\}
\end{aligned}
$$

The spin operators are expressed in terms of the singlet and triplet bond operators through the transformations given in Eqs.(3.3) and (3.4). The transformed Hamiltonian is given by

$$
\begin{aligned}
H & =\sum_{i}\left\{\sum _ { m = l , r } \left[\alpha\left(-\frac{3}{4} \mathbf{s}_{m i}^{\dagger} \mathbf{s}_{m i}+\frac{1}{4} \mathbf{t}_{m i \alpha}^{\dagger} \mathbf{t}_{m i \alpha}\right)\right.\right. \\
& -\mu_{m i}\left(\mathbf{s}_{m i}^{\dagger} \mathbf{s}_{m i}+\mathbf{t}_{m i \alpha}^{\dagger} \mathbf{t}_{m i \alpha}-1\right) \\
& +\frac{\lambda_{1}}{2}\left(\mathbf{t}_{m i \alpha}^{\dagger} \mathbf{t}_{m i+1 \alpha} s_{m i+1}^{\dagger} \mathbf{s}_{m i}+\mathbf{t}_{m i \alpha}^{\dagger} \mathbf{t}_{m i+1 \alpha}^{\dagger} \mathbf{s}_{m i+1} \mathbf{s}_{m i}+\text { H.C. }\right)
\end{aligned}
$$




$$
\begin{aligned}
& \left.-\frac{\lambda_{2}}{2} \epsilon_{\alpha \beta \gamma} \epsilon_{\alpha \beta^{\prime} \gamma^{\prime}} \mathbf{t}_{m i \beta}^{\dagger} \mathbf{t}_{m i+1 \gamma} \mathbf{t}_{m i \beta^{\prime}}^{\dagger} \mathbf{t}_{m i+1 \gamma^{\prime}}\right] \\
& +\frac{\lambda_{3}}{4}\left(\mathbf{s}_{l i}^{\dagger} \mathbf{t}_{l i \alpha} \mathbf{t}_{r i \alpha} \mathbf{s}_{r i}^{\dagger}+\mathbf{s}_{l i}^{\dagger} \mathbf{t}_{l i \alpha} \mathbf{t}_{r i \alpha}^{\dagger} \mathbf{s}_{r i}+H . C .\right) \\
& \left.-\frac{\lambda_{4}}{4} \epsilon_{\alpha \beta \gamma} \epsilon_{\alpha \beta^{\prime} \gamma^{\prime}} \mathbf{t}_{l i \beta}^{\dagger} \mathbf{t}_{l i \gamma} \mathbf{t}_{r i \beta^{\prime}}^{\dagger} \mathbf{t}_{r i \gamma^{\prime}}\right\}
\end{aligned}
$$

where

$$
\lambda_{1}=\beta-\gamma, \quad \lambda_{2}=\beta+\gamma, \quad \lambda_{3}=\eta-\xi, \quad \lambda_{4}=\eta+\xi
$$

where $\mathrm{m}$ denotes the ladder index, left (l) or right $(\mathrm{r})$ and $\mu_{\mathbf{m i}}$ is the chemical potential which has been introduced to take account of the constraint specified in Eq.(3.5). One takes the expectation value $\left\langle\mathbf{s}_{\mathbf{m i}}\right\rangle=\overline{\mathbf{s}}$ and the local chemical potential $\mu_{\mathbf{m i}}$ is replaced by the global one $\mu$. We perform a Fourier transformation of the operators $\mathbf{t}_{\mathbf{m i} \alpha}=\frac{\mathbf{1}}{\sqrt{\mathbf{N}}} \sum_{\mathbf{k}} \mathbf{t}_{\mathbf{m k} \alpha} \mathbf{e}^{-\mathbf{i k r} \mathbf{r}_{\mathbf{i}}}$ where N is the number of dimers or rungs in a two chain ladder and $\mathrm{k}$ is the wave vector along the ladder axis. The Fourier-transformed Hamiltonian is given by

$$
\begin{aligned}
H & =2 N\left(-\frac{3}{4} \alpha \overline{\mathbf{s}}^{2}-\mu \overline{\mathbf{s}}^{2}+\mu\right) \\
& -\frac{2}{3} \lambda_{2} N\left(P^{2}-Q^{2}\right)-\frac{1}{6} \lambda_{4} N\left(P^{\prime 2}-Q^{\prime 2}\right) \\
& +\sum_{k}\left[\sum_{m=l, r}\left(A_{k} \mathbf{t}_{m k \alpha}^{\dagger} \mathbf{t}_{m k \alpha}+B_{k}\left(\mathbf{t}_{m k \alpha}^{\dagger} \mathbf{t}_{m-k \alpha}^{\dagger}+\mathbf{t}_{m k \alpha} \mathbf{t}_{m-k \alpha}\right)\right)\right. \\
& \left.+C\left(\mathbf{t}_{l k \alpha}^{\dagger} \mathbf{t}_{r k \alpha}+\mathbf{t}_{r k \alpha}^{\dagger} \mathbf{t}_{l k \alpha}\right)+D\left(\mathbf{t}_{l k \alpha}^{\dagger} \mathbf{t}_{r-k \alpha}^{\dagger}+\mathbf{t}_{l k \alpha} \mathbf{t}_{r-k \alpha}\right)\right]
\end{aligned}
$$


where $A_{k}, B_{k}, \mathrm{C}$ and $\mathrm{D}$ are defined as

$$
\begin{aligned}
A_{k} & =\frac{\alpha}{4}-\mu+\left(\lambda_{1} \overline{\mathbf{s}}^{2}+\frac{2}{3} \lambda_{2} P\right) \cos k \\
B_{k} & =\left(\frac{1}{2} \lambda_{1} \overline{\mathbf{s}}^{2}-\frac{1}{3} \lambda_{2} Q\right) \cos k \\
C & =\frac{1}{4} \lambda_{3} \overline{\mathbf{s}}^{2}+\frac{1}{6} \lambda_{4} P^{\prime} \\
D & =\frac{1}{4} \lambda_{3} \overline{\mathbf{s}}^{2}-\frac{1}{6} \lambda_{4} Q^{\prime}
\end{aligned}
$$

$\mathrm{P}, \mathrm{Q}, P^{\prime}$ and $Q^{\prime}$ are the four mean-fields,

$$
\begin{gathered}
P=\left\langle\mathbf{t}_{m i \alpha}^{\dagger} \mathbf{t}_{m i+1 \alpha}\right\rangle, \quad Q=\left\langle\mathbf{t}_{m i \alpha} \mathbf{t}_{m i+1 \alpha}\right\rangle, \\
P^{\prime}=\left\langle\mathbf{t}_{r i \alpha}^{\dagger} \mathbf{t}_{l i \alpha}\right\rangle, \quad Q^{\prime}=\left\langle\mathbf{t}_{r i \alpha} \mathbf{t}_{l i \alpha}\right\rangle
\end{gathered}
$$

We now perform a Bogolyubov transformation into two new boson operators in terms of the $\mathbf{t}$ operators of the left and right hand ladders as

$$
\begin{aligned}
\tau_{1,2 k \alpha} & =\frac{1}{\sqrt{2}}\left[\left(\cosh \theta_{1,2 k} \mathbf{t}_{l k \alpha}+\sinh \theta_{1,2 k} \mathbf{t}_{l-k \alpha}^{\dagger}\right)\right. \\
& \left. \pm\left(\cosh \theta_{1,2 k} \mathbf{t}_{r k \alpha}+\sinh \theta_{1,2 k} \mathbf{t}_{r-k \alpha}^{\dagger}\right)\right]
\end{aligned}
$$

These are symmetric (bonding) and antisymmetric (antibonding) combinations of the transformations in the left and right ladders. The Hamiltonian (Eq.(3.15)) can now be diagonalised to obtain

$$
H_{m}\left(\mu, \overline{\mathbf{s}}, P, Q, P^{\prime}, Q^{\prime}\right)=2 N\left(-\frac{3}{4} \alpha \overline{\mathbf{s}}^{2}-\mu \overline{\mathbf{s}}^{2}+\mu\right)-N\left(\frac{\alpha}{4}-\mu\right)
$$




$$
\begin{aligned}
& -\frac{2}{3} \lambda_{2} N\left(P^{2}-Q^{2}\right)-\frac{1}{6} \lambda_{4} N\left(P^{\prime 2}-Q^{\prime 2}\right) \\
& +\sum_{k, m=1,2} \omega_{m k}\left(\tau_{m k}^{\dagger} \tau_{m k}+\frac{1}{2}\right)
\end{aligned}
$$

where $\omega_{1,2 k}$ is defined as

$$
\omega_{1,2 k}=\sqrt{\left(C \mp A_{k}\right)^{2}-\left(2 B_{k} \mp D\right)^{2}}
$$

The spin-triplet excitation spectrum of the four-chain ladder consists of two branches corresponding to the bonding and antibonding states. The magnitude of the splitting of the two branches is determined by $\lambda_{3}$ and $\lambda_{4}$. Thus the two branches collapse into a single branch, when both $\eta=0, \xi=0$ as in the case of a single two-chain ladder.

Eq. (3.19) describes the triplet excitation spectrum in a general parameter regime. The parameters $\mu, \overline{\mathbf{s}}, \mathbf{P}, \mathbf{Q}, \mathbf{P}^{\prime}$ and $\mathbf{Q}^{\prime}$ in the excitation spectrum are determined by solving the saddle-point equations:

$$
\begin{aligned}
& \left\langle\frac{\delta H_{m}}{\delta \mu}\right\rangle=0, \quad\left\langle\frac{\delta H_{m}}{\delta \bar{s}}\right\rangle=0, \quad\left\langle\frac{\delta H_{m}}{\delta P}\right\rangle=0, \\
& \left\langle\frac{\delta H_{m}}{\delta Q}\right\rangle=0, \quad\left\langle\frac{\delta H_{m}}{\delta P^{\prime}}\right\rangle=0, \quad\left\langle\frac{\delta H_{m}}{\delta Q^{\prime}}\right\rangle=0
\end{aligned}
$$

At $T=0$, the mean-field equations are obtained as

$$
\begin{aligned}
\overline{\mathbf{s}}^{2} & =\frac{3}{2}+\frac{1}{8 \pi} \int\left(\frac{C-A_{k}}{\omega_{\mathbf{1}}}-\frac{C+A_{k}}{\omega_{\mathbf{2}}}\right) d k \\
P & =-\frac{1}{8 \pi} \int\left(\frac{C-A_{k}}{\omega_{\mathbf{1}}}-\frac{C+A_{k}}{\omega_{\mathbf{2}}}\right) \cos k d k
\end{aligned}
$$




$$
\begin{aligned}
Q & =-\frac{1}{8 \pi} \int\left(\frac{2 B_{k}-D}{\omega_{1}}+\frac{2 B_{k}+D}{\omega_{\mathbf{2}}}\right) \cos k d k \\
P^{\prime} & =\frac{1}{8 \pi} \int\left(\frac{C-A_{k}}{\omega_{1}}+\frac{C+A_{k}}{\omega_{\mathbf{2}}}\right) d k \\
Q^{\prime} & =\frac{1}{8 \pi} \int\left(\frac{2 B_{k}-D}{\omega_{1}}-\frac{2 B_{k}+D}{\omega_{\mathbf{2}}}\right) d k \\
\mu & =-.75 \alpha+\lambda_{1}(P+Q)+\frac{\lambda_{3}}{4}\left(P^{\prime}+Q^{\prime}\right)
\end{aligned}
$$

Fig. 4 shows the spin-triplet excitation spectrum of the four-chain ladder for the exchange interaction strengths $\beta=2 \gamma=1$ and $\xi=2 \eta=1$, in units of $\alpha$. Fig. 5 shows the spin-gap of the four-chain ladder versus $\xi$ for $\beta=1$, $\eta=\frac{\xi}{2}$ and $\gamma=0.5$, in units of $\alpha$.

The ground state energy of the ladder system in the general parameter regime is given by

$$
\begin{aligned}
E_{g} & =2 N\left(-\frac{3}{4} \alpha \overline{\mathbf{s}}^{2}-\mu \overline{\mathbf{s}}^{2}+\mu\right)-N\left(\frac{\alpha}{4}-\mu\right)-\frac{2}{3} \lambda_{2} N\left(P^{2}-Q^{2}\right) \\
& -\frac{1}{6} \lambda_{4} N\left(P^{\prime 2}-Q^{\prime 2}\right)+\frac{1}{2} \sum_{k, m=1,2} \omega_{m k}
\end{aligned}
$$

The parameter regime includes the point $\alpha \geq 3 \beta, \beta=\gamma=\xi=\eta$ at which the ground state and the corresponding energy are exactly known. For these parameter values, $\lambda_{1}=\mathbf{0}, \lambda_{\mathbf{3}}=\mathbf{0}$ and $\lambda_{\mathbf{2}}=\mathbf{2} \beta, \lambda_{\mathbf{4}}=\mathbf{2} \beta$. Also, the four mean-fields $\mathbf{P}, \mathbf{Q}, \mathbf{P}^{\prime}, \mathbf{Q}^{\prime}$ are zero, $\overline{\mathbf{s}}^{2}=1$ and $\mu=-.75 \alpha$.

From Eq. (3.19), one then obtains a single excitation spectrum of energy $\omega_{\mathbf{k}}=\alpha$, i.e., the spectrum is dispersionless. The ground state energy $E_{g}$ 
becomes

$$
\begin{aligned}
E_{g} & =2 N\left(-\frac{3}{4} \alpha \bar{s}^{2}-\mu \bar{s}^{2}+\mu\right)-N\left(\frac{\alpha}{4}-\mu\right)+\frac{1}{2} \sum_{k, m=1,2} \omega_{\mathbf{m} \mathbf{k}} \\
& =-\frac{3}{2} \alpha N
\end{aligned}
$$

which is equal to the exact ground state energy. The mean-field theory based on the bond operator formalism thus reproduces the correct ground state energy in the appropriate limit of the coupling parameters.

Next we consider a periodic array of ladders, i.e., consider the full square lattice with exchange interactions as specified before. The problem of interest is to find the value of the interladder interaction strength $\xi$ at which the spin-gap disappears. For the usual square lattice $S=1 / 2$ HAFM with only n.n. interactions, long-range AFM order exists in the ground state and the spin-gap is expected to vanish at a critical value of the interladder exchange interaction $\xi(\eta=0, \gamma=0$ in this case $)$. The value obtained by Gopalan et al is $\xi=0.25$. The spin-ladder model constructed by us has not only n.n. but n.n.n. (along the rungs) as well as diagonal interactions. It is of interest to determine whether for this model also the spin-gap vanishes at a critical value of $\xi$. Using the formalism already developed, we obtain the self-consistent equations

$$
\begin{aligned}
\overline{\mathbf{s}}^{2} & =\frac{3}{2}+\frac{1}{8 \pi^{2}} \iint \frac{C_{k}-A_{k}}{\omega} d \mathbf{k} \\
P & =-\frac{1}{8 \pi^{2}} \iint \frac{C_{k}-A_{k}}{\omega} \cos k_{x} d \mathbf{k} \\
Q & =-\frac{1}{8 \pi^{2}} \iint \frac{2 B_{k}-D_{k}}{\omega} \cos k_{x} d \mathbf{k}
\end{aligned}
$$




$$
\begin{aligned}
P^{\prime} & =\frac{1}{8 \pi^{2}} \iint \frac{C_{k}-A_{k}}{\omega} \cos k_{y} d \mathbf{k} \\
Q^{\prime} & =\frac{1}{8 \pi^{2}} \iint \frac{2 B_{k}-D_{k}}{\omega} \cos k_{y} d \mathbf{k} \\
\mu & =-.75 \alpha+\lambda_{1}(P+Q)+\frac{\lambda_{3}}{2}\left(P^{\prime}+Q^{\prime}\right)
\end{aligned}
$$

where $A_{k}, B_{k}, C_{k}$, and $D_{k}$ are

$$
\begin{aligned}
& A_{k}=\frac{\alpha}{4}-\mu+\left(\lambda_{1} \overline{\mathbf{s}}^{2}+\frac{2}{3} \lambda_{2} P\right) \cos k_{x}, \\
& B_{k}=\left(\frac{1}{2} \lambda_{1} \overline{\mathbf{s}}^{2}-\frac{1}{3} \lambda_{2} Q\right) \cos k_{x} \\
& C_{k}=\left(\frac{1}{2} \lambda_{3} \overline{\mathbf{s}}^{2}+\frac{1}{3} \lambda_{4} P^{\prime}\right) \cos k_{y} \\
& D_{k}=\left(\frac{1}{2} \lambda_{3} \overline{\mathbf{s}}^{2}-\frac{1}{3} \lambda_{4} Q^{\prime}\right) \cos k_{y}
\end{aligned}
$$

Also, $\mathbf{k}$ is a two-dimensional wave vector with components $k_{x}$ (along the ladder axis) and $k_{y}$ (across the ladders). The excitation spectrum $\omega_{\mathbf{k}}$ is given by

$$
\omega_{\mathbf{k}}=\sqrt{\left(C_{k}-A_{k}\right)^{2}-\left(2 B_{k}-D_{k}\right)^{2}}
$$

The excitation spectrum $\omega_{\mathbf{k}}$ has a minimum at $\mathbf{k}=(\pi, 0)$. Fig. 6 shows a plot of the spin-gap $\Delta$ versus $\xi$ for $\beta=1, \gamma=0.5$ and $\eta=\frac{\xi}{2}$, in units of $\alpha$. The spin-gap $\Delta$ vanishes for $\xi=\mathbf{0 . 3 3}$. 


\section{Conclusions}

We have constructed spin ladders with odd and even number of chains for which in a particular parameter regime the exact ground state can be written down. It can further be shown rigorously that the excitation spectrum is gapless (with a gap) for odd (even) number of chains. The mean-field theory based on the bond operator formalism has been applied to ladders with an even number of chains in a general parameter regime. Both the formalism and the results obtained are similar to those of Gopalan et al $\mathbb{4}$ for spin ladders which differ from ours in that the diagonal and n.n.n. interactions along the rung are absent. One significant difference is in the inclusion of terms containing four triplet operators in our mean-field theory. For the ladder models considered by Gopalan et al these terms have a negligible contribution and so have been ignored. In the present case, the terms can no longer be neglected. The results of Gopalan et al [4] can be recovered from our results by putting $\gamma=\mathbf{0}, \eta=\mathbf{0}$. Inclusion of these extra interactions has the effect of renormalising the original coupling parameters of the Hamiltonian when expressed in terms of the singlet and triplet operators. For the fourchain spin ladder considered in Ref.4, the coupling parameters $\beta-\gamma$ and $\eta-\xi$ in $E q .(3.14)$ are $\beta$ and $-\xi$ respectively. The inclusion of frustrating further-neighbour interactions in our model has the effect of increasing the spin-gap. For the periodic ladder, the spin-gap vanishes at $\xi=0.33$ ( Fig. 6 ). The decrease of the spin-gap with $\xi$ is explained by the delocalisation of the singlets across the ladders. The decrease of the gap is faster than that of a four-chain ladder.

The mean-field theory based on the bond operator formalism reproduces the exact ground state energy in the appropriate limit. The same is true for 
two other spin models in 1d and 2d, namely, the Majumdar-Ghosh chain 18] and the $J_{1}-J_{2}-J_{3}-J_{4}-J_{5}$ model proposed by Bose and Mitra 19, 20.

The ground state of both the models can be determined exactly at particular values of the parameters. The ground states consist of a periodic arrangement of dimers. Mean-field theory based on the bond operator formalism determines the ground state energy correctly in the exactly-solvable limit. In the same limit, the mean-field theory yields a dispersionless excitation spectrum for both the spin models. This is also true for the ladder spin system signifying that the three spin models share common features.

The sawtooth chain which describes the exchange interactions along the rungs of the ladder system has been studied earlier by Kubo[17]. The ground state of the chain is doubly degenerate and the spin dynamics is described in terms of kink, antikink excitations.

Consider the parameter regime in which the exact ground state of the ladder system is known. The sawtooth chain interactions are now (Fig. 2) $\eta=\xi=\frac{\alpha}{3}$. In this case the ground state is nondegenerate with spin singlets forming along the stronger bonds. Kink, antikink excitations which can be considered as spin defects separating the two degenerate ground states are absent in this case. Spin excitations are now created if one of the singlets is replaced by a triplet and the triplet is allowed to propagate. In sawtooth chain with doubly degenerate ground states these excitations have a higher energy than the kink, antikink excitations.

\section{Acknowledgement}


We thank Sujit Sarkar for computational help. 


\section{Figure Captions}

Fig. 1 The two-chain ladder with rung, horizontal and diagonal exchange interactions of strength $\alpha, \beta$ and $\gamma$ and depicted by dashed ( bold ), solid ( bold ), and solid ( thin ) lines respectively .

Fig. 2 The sawtooth chain with three different interactons of strength $\alpha, \xi$ and $\eta$ and depicted by dashed ( bold ), dashed ( thin ) and dot-dashed lines respectively.

Fig. 3(a) The three-chain ladder with five different interactions of strength $\alpha, \beta, \gamma, \xi$ and $\eta$ and depicted by dashed ( bold ), solid ( bold ), solid ( thin ), dashed ( thin ) and dot-dashed lines respectively.

Fig. 3(b) The four-chain ladder with five different interactions of strength $\alpha, \beta, \gamma, \xi$ and $\eta$.

Fig. 4 The triplet excitation spectrum [ bonding and antibonding states of Eq. (3.19) ] of the four-chain ladder with exchange interaction strengths $\beta=2 \gamma=1$ and $\xi=2 \eta=1$, in units of $\alpha$.

Fig. 5 The spin-gap $\Delta$ of the four-chain ladder versus $\xi$, for $\eta=\frac{\xi}{2}, \beta=\mathbf{1}$ and $\gamma=\mathbf{0 . 5}$, in units of $\alpha$.

Fig. 6 The spin-gap $\boldsymbol{\Delta}$ of the peroidic ladder versus $\xi$, for $\eta=\frac{\xi}{2}, \beta=\mathbf{1}$ and $\gamma=\mathbf{0 . 5}$, in units of $\alpha$. The spin-gap vanishes for $\xi=\mathbf{0 . 3 3}$. 


\section{References}

[1] Elbio Dagotto and T. M. Rice, Science 271, 618 (1996); K. Takano, J. Phys. A: Math. Gen. 27, L269 (1994); T. Oguchi and H. Kitatani, J. Phys. Soc. Jpn. 64, 612 (1995); H. Kitatani and T. Oguchi, J. Phys. Soc. Jpn. 65, 1387 (1996).

[2] H. Bethe, Z. Phys. 71, 205 (1931).

[3] D. C. Johnston, J. W. Johnston, D. P. Goshorn and A. J. Jacobson, Phys. Rev. B 35, 219 (1987).

[4] S. Gopalan, T. M. Rice and M. Sigrist, Phys. Rev. B 49, 8901 (1994)

[5] M. Reigrotzki, H. Tsunetsugu and T. M. Rice, J. Phys.: Condens. Matter $\mathbf{6}, 9235$ (1994).

[6] D. V. Khveshchenko, Phys. Rev. B 50, 380 (1994).

[7] K. Hida, Phys. Rev. B 45, 2207 (1992); K. Hida and S. Takada, J. Phys. Soc. Jpn. 61, 1879 (1992); K. Hida, J. Phys. Soc. Jpn. 64, 4896 (1995).

[8] Steven R. White, Phys. Rev. B 53, 52 (1996).

[9] A. J. Rojo, Phys. Rev. B 53, 9172 (1996).

[10] S. Sachdev and R. N. Bhatt, Phys. Rev. B 41, 9323 (1990).

[11] Indrani Bose and Saurabh Gayen, Phys. Rev. B 41, 10653 (1993).

[12] Indrani Bose and Saurabh Gayen, J. Phys.: Condens. Matter 6, L405 (1994). 
[13] Saurabh Gayen and Indrani Bose, J. Phys.: Condens. Matter 7, 5871 (1995).

[14] Indrani Bose, Phys. Rev. B 45, 13072 (1992).

[15] J. des Cloizeaux and J. J. Pearson, Phys. Rev. 128, 2131 (1962).

[16] S. Gayen, I. Bose and A. Ghosh, to be published.

[17] K. Kubo, Phys. Rev. B 48, 10552 (1993).

[18] C. K. Majumdar, J. Phys. C 3, 911 (1970).

[19] I. Bose and P. Mitra, Phys. Rev. B 44, 443 (1991).

[20] U. Bhaumik and I. Bose, Phys. Rev. B 52, 12489 (1995). 